\title{
Scheduling of rehearsals with mixing piece length
}

\author{
Bin Liu \\ School of Management, Shanghai University, Shanghai 200444, China \\ lb_yjs@163.com
}

Keywords: Rehearsal, Mixing piece length, Genetic algorithm, Scheduling optimization.

\begin{abstract}
The cost of actors is one of the problem that have to be considered in the rehearsal. In traditional rehearsals, the piece length is fixed, but it is not always certain in practice. Based on the consideration of the actual situation, innovatively setting the piece length to mixing which consists of two parts, one part is the performance time which is determinate value; the other part is adjustment time for the rehearsal which is indeterminate value. The purpose of this study is to schedule the pieces reasonably in order to reduce the idle time of actors and minimize the total cost which is paid for the actors' waiting time. This paper presents a mixed integer programming model to optimize the sequence of the piece which has mixing length. For this model, we also designed a genetic algorithm based heuristic method to solve the problem in different scale, the test results demonstrate the effectiveness of this method.
\end{abstract}

\section{Introduction}

A successful performance appearing in front of the audience needs perfect rehearsal. In the rehearsal, how to scheduling the program in order to reduce the total costs for the actors' waiting time is one of the main issues to consider. This problem is actually very similar to film shooting, workshop and project scheduling which aim to minimize the cost by reasonable scheduling [1]. Generally speaking, there are two different ways of paying for every actor's performance fee: one is that each actor has the same fee; another is different. Allowing for scale and efficiency, the rehearsal time can be divided into two ways: continuous and discrete. Continuous requires that the rehearsals should start from the first piece until the last one to be completed, in addition, no interruption in the process $[2,3]$. For the rehearsals which have large scale and will take long time, the common choice is discrete. In this situation, rehearsals may take several days and each day of rehearsal time is fixed. When rehearsal time is running out in that day, it will arrange rehearsal to the next day [4]. For each program, the required length of time rehearsing are generally assumed to determine the value.

\section{Model formulation}

\author{
Notations \\ Indices and parameters: \\ $T \quad$ index of time series, $T=1,2, \cdots, m$; Each piece occupies a time series. The time series \\ are arranged in ascending order. \\ $S \quad$ index of pieces, $S=1,2, \cdots, m$. \\ $A \quad$ index of actors, $A=1,2, \cdots, q$. \\ $C \quad$ index of actors' unit cost, $C=c_{1}, c_{2}, \cdots, c_{q}$. \\ $F \quad$ sets of the probability of scenario, $F=p_{1}, p_{2}, \cdots, p_{w}$. \\ W number of scenarios. \\ $D \quad$ sets of the length of pieces, $D=d_{1}+n(f)_{1}, d_{2}+n(f)_{2}, \cdots, d_{m}+n(f)_{m}$. $d$ represents \\ the performance time; $n(f)$ represents the adjustment time under scenario $f$
}


$u_{a s} \quad 1$ if actor $a$ plays piece $s, 0$ otherwise.

Decision variables:

$x_{a t} \quad 1$ if actor $a$ plays in time series t. 0 otherwise.

$y_{s t} \quad 1$ if piece $s$ is rehearsed in time series $t .0$ otherwise.

$v_{a t} \quad 1$ if actor $a$ is on the rehearsal scene in time series $t .0$ otherwise.

$r_{a t} \quad 1$ if actor $a$ arrives in or before time series $t .0$ otherwise.

$l_{a t} \quad 1$ if actor $a$ leaves in or after time series $t .0$ otherwise.

\section{Mathematical model}

As mentioned above, the objective is to minimize the total waiting cost of all the actors:

Minimize $Z=\sum_{s=1}^{m} \sum_{a=1}^{q} \sum_{t=1}^{m} d_{s} \cdot c_{a} \cdot w_{a t}+\sum_{f=1}^{w} \sum_{s=1}^{m} \sum_{a=1}^{q} \sum_{t=1}^{m} p_{f} \cdot n(f)_{s} \cdot c_{a} \cdot w_{a t}$

s.t. $\sum_{s=1}^{m} y_{s t}=1, \forall t \in T$

$\sum_{t=1}^{m} y_{s t}=1, \forall s \in S$

$x_{a t}=\sum_{s=1}^{m} y_{s t} \times u_{a s}, \forall a \in A, \forall t \in T$

$v_{a t} \geq r_{a t}+l_{a t}-1, \forall a \in A, \forall t \in T$

$r_{a t} \leq \mathrm{r}_{a(t+1)}, \forall a \in A, \forall t \in\{1,2, \ldots, n-1\}$

$l_{a t} \geq l_{a(t+1)}, \forall a \in A, \forall t \in\{1,2, \ldots, n-1\}$

$r_{a t} \geq x_{a t}, \forall a \in A, \forall t \in T$

$l_{a t} \geq x_{a t}, \forall a \in A, \forall t \in T$

$v_{a t}-w_{a t} \leq x_{a t}, \forall a \in A, \forall t \in T$

The objective function (1) is to minimize the total waiting cost of all the actors. The objective function consists of two parts: one part is waiting cost under performance time which equals unit performance fee multiplied by waiting time. The other part is waiting cost under adjustment time which equals unit performance fee multiplied by waiting time by the probability of scenarios. Constraints (2) ensures that exactly one piece is rehearsed in each time series. Constraint (3) ensures that each piece can only appear in only one time series. Constraint (4) assign actors to the time series that they are required to play. Constraint (5-7) determine the presence of each actors in each time series. Constraint (8) ensures that each actor will arrive at the beginning of the first piece that he involves in. Constraint (9) ensures that each actor will leave at the end of the last piece that he involves in. Constraint (10) determine whether time series t is waiting time of actor $a$ or not.

\section{Numerical experiments}

\section{The steps of experimental}

First of all, set the number of pieces and actors. For example, there are 20 pieces requiring to be rehearsed and the 20 pieces need 40 actors.

Next, set the variance of the adjustment time of each piece. Since the adjustment time is uncertain, we can make the adjustment time obey normal distribution function [5]. It is assumed that the mean value is 2 and the variance is $1 、 1.5 、 2$. The discrete degree of data can be adjusted by changing the variance.

Then, set the crossover probability and mutation probability. Both of them need to be determined by the numerical experiments [6]. The result shows that the convergence effect is the best when Pc is 0.9 and $\mathrm{Pm}$ is 0.05 . 
Finally, introduce the parameter - scenario. We need figure out the best value of scenario. 40 pieces, 20 actors is the condition. The variance of adjustment time is $1, \mathrm{Pc}$ is 0.9 and $\mathrm{Pm}$ is 0.05 . Scenario has four different values: 10、30、50、100. Run the code 10 times and figure out the optimal solution under different scenarios. The test results as shown in Table 1:

Table 1 Experiments of the proposed model under different numbers of scenarios

\begin{tabular}{ccccccc}
\hline $\begin{array}{c}\text { Number of } \\
\text { Scenarios }\end{array}$ & Avg. & S.D. & Max. & Min. & $\begin{array}{c}\text { Gap } \\
\text { Max-Min }\end{array}$ & $\begin{array}{c}\text { Avg. CPU } \\
\text { Time (s) }\end{array}$ \\
\hline 10 & 16005.80 & 189.94 & 16387 & 15645 & 742 & 233 \\
30 & 15983.90 & 176.17 & 16356 & 15725 & 631 & 628 \\
50 & 15981.40 & 150.39 & 16352 & 15786 & 566 & 1053 \\
100 & 15978.50 & 139.06 & 16326 & 15837 & 489 & 2542 \\
\hline
\end{tabular}

In order to make the results more realistic, meanwhile increase the efficiency of the algorithm, this paper Set the number of scenarios to 30 .

After determining the above parameters, we consider the following two situations: first, the rehearsal time of pieces is determined value; second, the rehearsal time of the piece is uncertain value. In order to ensure the validity of test data, we can use the results of the different scale of the problem. Scale of the problem can be set to three conditions: 10 pieces、 20 actors, 15 pieces 30 actors, 20 pieces、 40 actors. Run the genetic code directly and figure out the optimal solution when the rehearsal time is certain. If the rehearsal time is uncertain, we should find the optimal solution under each scenario, and then calculate the average.

Finally, compare the test data. Compare the test data under the two conditions by changing the scale of the problem. Compare the test data under the two conditions by changing the variance of the adjustment time in the same scale.

\section{Analysis of result}

This experiment adopt the improved genetic algorithm. The initial population size is set to 50, the largest number of iterations is set to 100 . Using Matlab software to program and compute. The test data as shown in Table 2.

Comparing Z1 with Z2 under any scale, we can find that the waiting cost under uncertain rehearsal time is always smaller than the waiting cost under certain rehearsal. This shows that the proposed model and optimization algorithm is suitable for the problem of piece scheduling under any scale. What is more, the larger the scale of the problem is, the much cost advantage the proposed model will have.

In the same scale, increasing with the variance of adjustment time, $\mathrm{Z} 2$ is always bigger than $\mathrm{Z} 1$. In addition, (Z2-Z1) increases too. For example, under the scale of "40_20", when the variance of adjustment time growths from 1 to 2, (Z2-Z1) growths from 169.8 to 206.0. It shows that the proposed optimization algorithm has much more cost advantage comparing with the traditional method when the variance of adjustment time increases.

In addition, comparing Z1 with Z2 under the same variance of adjustment time and different scale of problem, we can draw a conclusion that the value of (Z2-Z1) increase with the scale of problem. For example, under the scale of " $20 \_10$ " and "30_20" , setting the variance of adjustment time to 1.5, (Z2-Z1 )growths from 48 to 186.7. Under the scale of " $30 \_15$ ” and " $40 \_20$ ”, setting the variance of adjustment time to 2, (Z2-Z1) growths from 94.1 to 206.0. It shows that the proposed optimization algorithm has much more cost advantage comparing with the traditional method when the scale of problem increases. 
Table 2 Comparisons between the certain rehearsal time and the uncertain rehearsal time

\begin{tabular}{ccccc}
\hline $\begin{array}{c}\text { Scale } \\
\text { actors_pieces }\end{array}$ & $\begin{array}{c}\text { Variance of } \\
\text { adjustment } \\
\text { time }(\mu)\end{array}$ & $\begin{array}{c}\text { Total waiting cost } \\
\text { (uncertain rehearsal } \\
\text { time)Z1 }\end{array}$ & $\begin{array}{c}\text { Total waiting cost } \\
\text { (certain rehearsal } \\
\text { time)Z1 }\end{array}$ & $\begin{array}{c}\text { Cost } \\
\text { reduction } \\
(\text { Z2-Z1 })\end{array}$ \\
\hline \multirow{2}{*}{ 20_10 } & 1 & 1645.8 & 1654.9 & 9.1 \\
& 1.5 & 1712.9 & 1760.9 & 48.0 \\
\hline $30 \_15$ & 2 & 1721.6 & 1799.2 & 77.6 \\
\hline \multirow{2}{*}{ 40_20 } & 1 & 6801.9 & 6822.7 & 20.8 \\
& 1.5 & 6999.1 & 7077.2 & 78.1 \\
& 1 & 6825.5 & 6919.6 & 94.1 \\
\hline
\end{tabular}

\section{Summary}

In this paper, due to the waiting time in the rehearsal, the study objective is to minimize the total waiting cost of all the actors by reasonable scheduling. We innovatively divide the rehearsal time into two parts: performance time and adjustment time. The performance time is determined value and the adjustment time is undetermined value. In this study, a mixed integer programming model is built. A heuristic algorithm based on genetic algorithm is designed to solve the model effectively. Meanwhile, compare the proposed optimization method with the traditional method which assumes that the rehearsal time is certain by a large number of experiments. From the result of the experiments, we can reach decisions that the waiting cost of the proposed optimization algorithm is always smaller than the traditional method, the proposed optimization algorithm has much more cost advantage comparing with the traditional method when the variance of adjustment time increases, the proposed optimization algorithm has much more cost advantage comparing with the traditional method when the scale of problem increases. Using the developed optimization method can solve the problem of rehearsal scheduling when the length of piece is uncertain. Moreover, it can be used as the reference when the organizers make scheduling scheme.

\section{References}

[1] Cheng T C E, Diamond J E, Lin B M T. (1993). Journal of Optimization Theory and Applications, 79(3), 479-492.

[2] Sakulsom N, Tharmmaphornphilas W. (2014). Computers \& Industrial Engineering, 70, 20-30.

[3] Bomsdorf F, Derigs U. (2008). Or Spectrum, 30(4), 751-772.

[4] Nordström A L, Tufekci S. (1994). Computers \& Operations Research, 21(8), 927-940.

[5] Garcia De La Banda M, Stuckey P J, Chu G. (2011). INFORMS Journal on Computing, 23(1), $120-137$.

[6] Kochetov Y. (2011) Iterative local search methods for the talent scheduling problem//Proceedings of 1st international symposium and 10th Balkan conference on operational research, September 22, Thessaloniki, Greece. 282-288. 